\title{
Underexpanded Supersonic Plasma Jet Generated by a Small Arc-Heated Facility
}

\author{
Heji Huang, Wenxia Pan, and Chengkang Wu
}

\begin{abstract}
As a flexible and efficient heat source to generate high enthalpy flows, a small arc-heated facility is unique in its ability to reproduce local aerodynamic heating environments for a long time period, to be used for validating the survivability of thermal protection materials used in hypersonic flights. Images of an underexpanded supersonic plasma jet generated by a small arc-heated facility and its interaction with some typical material samples are presented.
\end{abstract}

Index Terms-Fluid dynamics, plasma sources, supersonic jet.

$\mathbf{T}$ HE INTERACTIONS between supersonic jets and the structure/material on which they impinge are of interest in astronautic and aeronautic processes. Many studies on this topic have been carried out both experimentally [1], [2] and theoretically [3], [4]. However, since the flow fields in these situations are generally quite complex, often containing a mixture of supersonic and subsonic regions and shock interactions, to describe the details of the flow structure is still difficult. Moreover, when such jets are used for materials testing, the performance of specific materials often cannot be clearly defined if the structure of the flow field is not well understood.

In the design and testing of novel thermal protection materials, it is important to reproduce the aerothermodynamic environment that the material might withstand and to study the corresponding properties of materials. Small-scale plasma testing facilities, owing to their flexibility, simplicity, good controllability, and cost efficiency, are attractive in such studies. It is convenient to generate plasma with different gas compositions, velocities, and temperatures.

A $150-\mathrm{kW}$ arc-heated plasma testing facility built in the Institute of Mechanics, Chinese Academy of Sciences, was used to generate underexpanded supersonic plasma jets. The convergent-divergent conical nozzle has a 17-mm exit diameter $D$, with a design Mach number of 3.3. The nozzle was connected to a vacuum chamber. Nitrogen was used as the plasma gas with a flow rate of $60 \mathrm{slm}$, and the corresponding chamber pressure was $400 \mathrm{~Pa}$ during the experiment. This results in a nozzle-exit-pressure-to-chamber-pressure ratio $\left(P_{r}\right)$ of four. A

Manuscript received November 30, 2010; accepted May 1, 2011. Date of publication June 16, 2011; date of current version November 9, 2011. This work was supported by the National Natural Science Foundation of China under Grant 50702064 and 10921062.

The authors are with the Institute of Mechanics, Chinese Academy of Sciences, Beijing 100190, China (e-mail: huang@imech.ac.cn; wxpan@imech. ac.cn; ckw@imech.ac.cn).

Digital Object Identifier 10.1109/TPS.2011.2153214 molybdenum rod $2 \mathrm{~mm}$ in diameter placed across the jet and a copper flat plate $60 \mathrm{~mm}$ in diameter normal to the jet were used as typical sample shapes, and pictures were taken to show the flow field structures of the impingement of the supersonic jet on these objects. With varying brightness of the plasma, the qualitative structure of the flow field can be seen directly in the photographs taken by a Nikon D90 camera with a $60-\mathrm{mm}$ f/2.8D AF Micro-Nikkor lens.

Fig. 1(a) shows part of the underexpanded free plasma jet. A typical pattern of underexpanded supersonic jets is clearly seen. The expansion wave from the nozzle exit reflects from the constant pressure jet boundary (region 1) as compression wave and subsequently coalesces to form a weak barrel shock in region 2. The barrel shock then terminates in a triple point where a strong shock of Mach disk configuration forms (region 3). The weak barrel shock and the strong shock then merge together, giving birth to a reflected oblique shock (region 4). The subsonic region bounded by slip lines under the Mach disk cannot be distinguished in such a direct image, while it might be seen in a shadowgraph. In the free underexpanded supersonic plasma jet, such a pattern composed of the weak, strong, and reflected shocks is repeated to downstream with the total visible length of the jet longer than $600 \mathrm{~mm}$. The size of the expansion-compression cell depends on the ratio of the nozzle exit pressure and the chamber static pressure. With $P_{r}$ of four, the first Mach disk is far downstream in the supersonic jet at the relative axial position of $z / D$ of six to seven. Here, $z$ represents the axial position, and $D$ is the diameter of the nozzle exit. The succession of cells is the primary mechanism by which the pressure in the jet adapts to finally reach the ambient state.

When a thin molybdenum rod is inserted crosswise into the first cell of the plasma jet, a bow shock forms in front of it. Fig. 1(b) shows the end-on view of the rod (the small bright spot just above the numeral 6). The downstream region of the bow shock is a subsonic region, indicated by numeral 7 . The thin bright region (8) under the rod is a wake colored by a slight ablation of the molybdenum. The reflected shock in Fig. 1(a) has been pushed downstream to the position of $z / D$ of about eight to nine, indicated by numeral 9 in Fig. 1(b). Fig. 1(c) shows the impingement of the underexpanded supersonic plasma jet on a flat plate. Because the plate is positioned in the first cell of the jet at the position of $z / D=4$, the strong parameter changes, including those of velocity, pressure, and temperature of the flow near the plate surface, forming a plate shock. The bright subsonic region under the shock (11) is clearly seen in Fig. 1(c).

In summary, images of an underexpanded supersonic plasma jet generated by a small arc-heated testing facility have been 


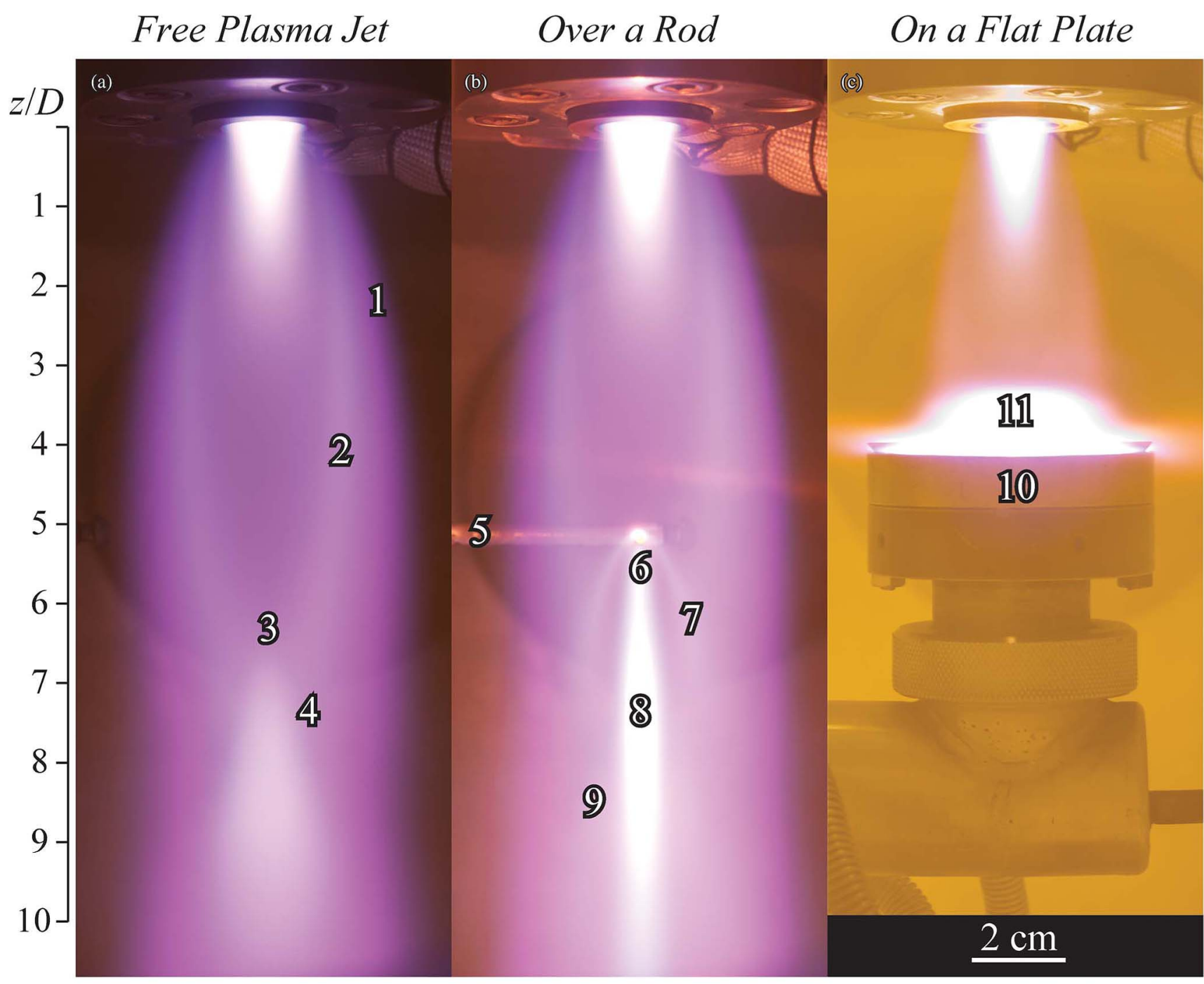

Fig. 1. (a) Underexpanded supersonic plasma jet and its impingement (b) over a thin molybdenum rod and (c) on a flat plate. (1) Constant pressure boundary. (2) Barrel shock. (3) Mach disk. (4) Reflected shock. (5) Support of the rod. (6) End of the rod. (7) Bow shock boundary. (8) Ablation product. (9) Reflected shock. (10) Water-cooled copper plate. (11) Recirculation zone.

presented. The pictures of impingement of such a plasma jet over a thin rod and on a flat plate are also shown.

\section{REFERENCES}

[1] L. F. Henderson, "Experiments on the impingement of a supersonic jet on a flat plate," Zeitschrift für Angewandte Mathematik und Physik (ZAMP), vol. 17, no. 5, pp. 553-569, Sep. 1966.
[2] Y. Nakai, N. Fujimatsu, and K. Fujii, "Experimental study of underexpanded supersonic jet impingement on an inclined flat plate," AIAA J., vol. 44, no. 11, pp. 2691-2699, Nov. 2006.

[3] C.-W. Chu, S. A. Powers, and H. Ziegler, "On the impingement of a supersonic jet on a flat plate," Zeitschrift f 'r Angewandte Mathematik und Physik (ZAMP), vol. 20, no. 1, pp. 15-18, Jan. 1969.

[4] A. Dauptain, B. Cuenot, and L. Y. M. Gicquel, "Large eddy simulation of stable supersonic jet impinging on flat plate," AIAA J., vol. 48, no. 10, pp. 2325-2338, Oct. 2010. 